\title{
Escala de estratégias de leitura para etapa inicial do ensino fundamental
}

\section{Reading strategy scale for elementary school students}

\author{
Maria Cristina Rodrigues Azevedo JOLY
}

\begin{abstract}
Resumo
Este estudo teve por objetivos verificar evidências de validade e precisão de uma escala de estratégias metacognitivas de leitura para crianças na fase inicial de escolarização. Participaram do presente estudo 1259 sujeitos com idade entre 9 e 14 anos que freqüentavam regularmente de $2^{a}(19,80 \%)$ a $4^{a}(50,00 \%)$ série do ensino fundamental. Pertenciam à escola particular 39,80\% e $61,20 \%$ a escolas públicas. Dos participantes, 51,00\% eram do sexo masculino. O procedimento utilizado foi a aplicação coletiva do instrumento Escala de Estratégias de Leitura - nível fundamental I. Os resultados revelaram que a escala pode ser considerada fidedigna $(\alpha=0,73)$. A análise fatorial indicou a presença de três fatores - estratégias globais de solução de problemas e de apoio - que explicam $42,12 \%$ da variabilidade da escala. Foram eliminados quatro itens por possuírem carga fatorial abaixo de 0,30, contando a nova versão da escala com 13 itens. A Anova indicou diferença muito significativa para utilização das estratégias de leitura por série e o teste " $t$ " de Student para amostras independentes revelou efeito do gênero para a freqüência total de uso da escala de estratégias de leitura, sendo essas evidências de validade de critério.
\end{abstract}

Palavras-chave: psicometria; validação, leitura; metacognição.

\begin{abstract}
This study has aimed the validation of a scale of metacoginitive reading strategies for children on their first stage at school. 1.259 students, aged from 9 to 4, took part in this study. All of them attended the elementary school, 39.80\% from private and $61.20 \%$ from public schools. Among the subjects, $51.00 \%$ were male. The Reading Strategies Scale-Primary School 1 was applied in groups. The results showed that the scale can be considered trustworth $(\alpha=0.73)$. Moreover, the factorial analysis revealed the existence of threefactors-global strategies, problem-solving and support - which guarantees this scale variability in $42.12 \%$. Four itens were eliminated because they presented scores below 0.30. There are thirteen-items in the new scale. According to the statistic analysis, there was a significant difference among students' grades about using the reading strategies ( $f=3.297 ; p=0.000 ; \alpha=0.05)$. The " $t$ "Student test for independent samples indicated gender influence in the total frequency of the reading strategies scale use $(t=5.266 ; p=0.000)$, which is a criteria for validation.
\end{abstract}

Key words: psychometria; validity, reading; metacognition.

A metacognição ou gerenciamento de metas, na perspectiva cognitiva do processamento humano da informação, coordena e monitora as atividades mentais. Para Veenman, Wilhelm e Beishuizem (2004), a metacognição é tanto uma habilidade geral, característica do desenvolvimento dos indivíduos, quanto específica, por ter características estratégicas em função da tarefa a ser realizada de estar parcialmente relacionada à inteligência.

\footnotetext{
$\boldsymbol{\nabla \nabla \nabla \nabla}$
}

1 Professora Doutora, Curso de Graduação e Pós-Graduação stricto sensu em Psicologia, Universidade São Francisco. Rua Alexandre Rodrigues Barbosa, 45, 13251-900, Itatiba, SP, Brasil. E-mail: <cristina.joly@saofrancisco.edu.br>. 
No que tange à leitura, estudos internacionais desenvolvidos por Flavell (1979), Moktari e Reichard (2002) e Ruíz Bolívar (2002) por exemplo, verificaram que há procedimentos metacognitivos que auxiliam a resolução de problemas de compreensão denominados estratégias de leitura, que permitem ao leitor compreender um texto com maior eficácia por possibilitarem o planejamento, a monitoração e a regulação dos próprios processos cognitivos envolvidos nessa tarefa.

Essas estratégias utilizadas para melhorar a compreensão da leitura, segundo González Fernández (1992), possibilitam ao leitor identificar os objetivos da leitura, destacar os aspectos importantes do texto e, especialmente, verificar se está havendo compreensão. Para tanto, o leitor deve utilizar as estratégias para facilitar as atividades de gerenciamento da leitura e providenciar ações corretivas quando se detectar falhas na compreensão. A literatura científica da área revela evidências de relações existentes entre treinamentos de estratégias de leitura e melhor desempenho na habilidade de compreensão (Morles, 1986).

As estratégias de leitura devem ser selecionadas considerando-se a maturidade do leitor, a abordagem e a complexidade do texto, o lugar onde o leitor se encontra na frase ou no texto, o momento da leitura e o seu propósito, a fim de possibilitar ao leitor efetuar um plano para compreender o texto (Kopcke, 1997; 2001). As estratégias mais freqüentemente utilizadas, segundo Pearson e Camperell (2001), foram classificadas em: globais, quando se referem à análise do texto de forma geral; de suporte, quando o leitor usa materiais de referências, grifos, dentre outros, para compreender o texto; de solução de problemas, quando surgem dificuldades de compreensão para o leitor diante de informações presentes no texto.

Rhoder (2002) em seu estudo confirma a possibilidade de ensinar os alunos a usarem deliberadamente estratégias para ler e entender o texto, de forma implícita e explícita. Para tanto, os alunos necessitam conhecer quais estratégias podem usar, como, quando, onde e por quê. Para que isso ocorra é necessário que aprendam as estratégias em situações educacionais, desde o início da escolarização.

\section{Estratégias de leitura e leitores iniciantes}

Pinnell, Lyons, DeFord, Bryk e Seltzer (1994)

272 sugerem que quando as crianças estão aprendendo a ler, adquirem um esquema de operação mental que as ajuda a elaborar um sistema de leitura individual, que contém estratégias de leitura específicas que as crianças iniciantes usam para significar e compreender como elas lêem. Existem muitas evidências, levantadas em pesquisas, que indicam que crianças aprendem usar diferentes estratégias de leitura para dar significado às palavras enquanto desenvolvem competência em leitura (Juel, 1980; Mclntyre, 1990; Sulzby, 1985; Taylor, Wade \& Yekovich, 1985).

No que se refere ao uso de estratégias de leitura por crianças, Collins (1998), em estudo realizado com doze crianças norte-americanas de cinco a sete anos, identificou oito estratégias utilizadas por elas no início da aprendizagem de leitura. As estratégias indicam como as crianças respondem às figuras e às palavras, como usam a memória e o som. O estudo mostra como professores podem usar os conhecimentos sobre as estratégias mais utilizadas por seus alunos para desenvolver os planos de aula, visando a uma melhor compreensão.

Em um estudo desenvolvido com objetivo de investigar a participação de crianças em um programa de leitura usando vídeo-cassete, observou-se que elas demonstraram uma ampliação no uso de estratégias metacognitivas. Tais estratégias correspondiam aos tipos usados por crianças mais experientes (Juliebo, Malicky \&Norman, 1998).

Dois estudos realizados em escolas públicas e privadas brasileiras demonstraram a eficácia de treinamento em uso de estratégias com crianças. Um desses estudos, realizado por Dias, Morais e Oliveira (1995), analisou a relação entre o nível de compreensão de leitura (muita dificuldade, média dificuldade e pouca dificuldade) da criança e o grau de benefício proporcionado pelo uso de estratégia de leitura. As crianças que participaram do programa de ensino de estratégias metacognitivas de leituras melhoraram significativamente a compreensão de textos em relação aos níveis apresentados anteriormente. O mesmo não ocorreu com as crianças do grupo-controle.

Em outro estudo realizado por Ferreira e Dias (2002), as crianças foram divididas em três grupos - dois experimentais e um controle. Um dos 
grupos experimentais utilizou a atividade de tomar notas; o outro, estratégia de imagem mental; e o grupocontrole não usou estratégia. A utilização dos dois tipos de estratégias possibilitou um melhor desempenho dos grupos experimentais ao responderem questões inferenciais e literais de compreensão em relação ao grupo-controle. O grupo que usou a estratégia de tomar notas, e que possuía muita dificuldade em compreensão, progrediu mais sobre questões de inferência do que o outro que apresentava menos dificuldade de compreensão.

Considerando-se a revisão da literatura (Brown, Pressley, van Meter \& Schuder, 1996; Dembo, 2000; Lorch, Lorch \& Klusewitz,1993; Palincsar \& Brown, 1984; Song, 1998; Vicentelli, 2000; Williams, 2002) pode-se constatar que há, por um lado, uma preocupação com o ensino de estratégias de leitura. Por outro, não há instrumentos validados no Brasil que possam avaliar especificamente tais estratégias, considerando-se os diferentes momentos da leitura. Faz-se necessário que instrumentos sejam desenvolvidos para esse fim com as qualidades psicométricas que devem possuir a fim de que sejam consistentes e reconhecidos do ponto de vista científico (Joly \& Noronha, 2004).

Um dos graves problemas que se tem enfrentado na avaliação psicológica, segundo Noronha (2002), é a falta de rigor científico dos instrumentos padronizados que são usados no Brasil. No tocante às estratégias de leitura há no Brasil uma escala de avaliação criada por Joly (2004), destinada a verificar a freqüência de uso por universitários, que se mostrou com boa precisão $(\alpha=0,91)$ e com evidência de validade de construto obtida por meio de análise fatorial que identificou os fatores de suporte à leitura, global e solução de problemas (Joly, Cantalice \& Vendramini). Não se têm descrito na literatura estudos relativos a instrumentos para leitores iniciantes.

Considerando-se, pois, os aspectos anteriormente abordados no tocante à necessidade de instrumentos para avaliar o processo de compreensão em leitura e a importância das estratégias metacognitivas como facilitadoras da compreensão, esse estudo tem por objetivos verificar evidências de validade e precisão de uma escala de estratégias metacognitivas de leitura para crianças na fase inicial de escolarização.

\section{Método}

\section{Participantes}

Participaram do presente estudo 1259 sujeitos com idade entre 7 e 14 anos (IM=9,31; $D P=1,09) ; 0,4 \%$ tinha sete anos, 89,3\% entre oito e dez anos e 10,3\% acima de dez anos. Freqüentavam regularmente de $2^{\text {a }}$ $(19,8 \%)$ a $4^{a}(50,0 \%)$ série do ensino fundamental. Dos alunos que estavam na $2^{a}(n=249)$ e $3^{a}$ série $(n=376)$, 94,0\% tinham entre sete e nove anos; desses, 65,1\% com oito anos estavam na $2^{\text {a }}$ e $47,1 \%$ na $3^{\text {a }}$ série. Na $4^{a}$ série ( $\left.n=634\right)$, 95,6\% tinham entre 9 e 11 anos. Pertenciam à escola particular 39,8\% e 61,2\% a escolas públicas de cidades do interior paulista. Dos participantes, 51,0\% eram do sexo masculino.

\section{Instrumento}

A Escala de Estratégias de Leitura (EELI), nível fundamental I, tem por objetivo avaliar o tipo e a freqüência de estratégias metacognitivas que as crianças utilizam, antes, durante e após a leitura de textos informativos. É composta por 17 afirmações do tipo Likert com três pontos (nunca: 0 ponto; algumas vezes: 1 ponto; sempre: 2 pontos), divididas em três categorias, que apontam os três fatores relacionados às estratégias. O fator 1 inclui dez estratégias metacognitivas globais - utilizadas para análise geral do texto (questões $1,2,3,4,5,6,7,8,9$ e 14). O fator 2 inclui quatro estratégias metacognitivas de suporte à leitura - são as denominadas funcionais ou de apoio que envolvem o uso de materiais de referências e/ou anotações (questões 11, 12, 13 e 17). O fator 3 inclui três estratégias metacognitivas de solução de problemas - usadas frente a dificuldades específicas de compreensão (questões 10, 15 e 16). A somatória da pontuação pode ser feita por fator, por momento de leitura e considerando-se todos os itens.

Esse instrumento desenvolvido por Joly (2004) foi baseado em Reading Strategies I Use: Intermediate (Hil, Rupti \& Norwick, 1998); Motivations For Reading Questionnaire, Revised Version (Guthrie \&Wigfielg, 1999), Reading Strategy Cheklist (Harp, 2000), A Questionnare to Measure Children's Awareness of Strategic Reading Process (Schmidt, 2000) e Assessing Students Metagocnitive 
Awareness of Reading Strategies (Mokhtari \& Reichard, 2002). Optou-se por utilizar como referências esse material americano devido à inexistência de correlatos no Brasil. Não há informações sobre características psicométricas do instrumento, pois essa análise será objeto do presente estudo.

\section{Procedimentos}

O instrumento foi aplicado, de forma coletiva, em uma sessão com duração de aproximadamente 30 minutos. Seguindo as instruções do aplicador que leu cada item e esperou que os sujeitos assinalassem a resposta escolhida, os sujeitos assinalaram a freqüência com que utilizam as estratégias de leitura quando lêem textos do tipo informativo. As aplicações foram feitas por escola, série e classe, de acordo com agendamento prévio com a direção de cada uma.

\section{Resultados e Discussão}

Verificando os pressupostos para efetuar a análise fatorial, o teste de Esfericidade de Bartlett indicou que a matriz de correlação não é uma matriz identidade, portanto existe correlação entre os itens avaliados $\left(\chi^{2}[136, n=1259]=2565,631 ; p<0,001\right)$, e a medida de adequação da amostra de Kaiser-Meyer-Olkin $(\mathrm{KMO}=0,865)$ revelou que a amostra é adequada para avaliar as estratégias de leitura utilizadas pelos sujeitos, conferindo validade aos resultados.

A partir da análise fatorial completa, dos valores extraídos do gráfico scree plot (Figura 1), do critério de raiz latente que considera significantes apenas os autovalores maiores que um e do número de fatores estabelecidos inicialmente na teoria (Mokhtari \& Reichard, 2002), optou-se por considerar quatro fatores que explicavam 41,89\% da variância total e três fatores.

Analisando as cargas fatoriais e as comunalidades da matriz inicial de componentes principais com quatro e três fatores (nessa ordem), conforme sugere o gráfico scree plot (Figura 1), observou-se que a melhor configuração explicável pela teoria e mais próxima da

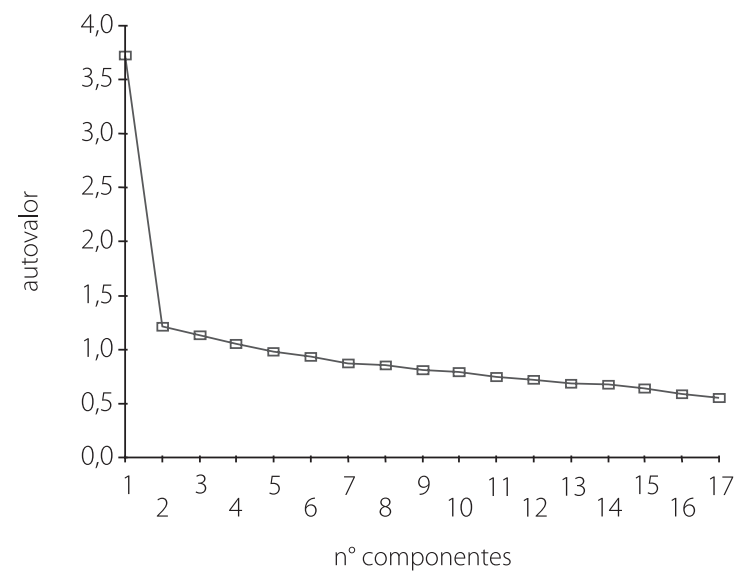

Figura 1. Resultado da análise dos itens pelo método scree plot.

Tabela 1. Valor de comunalidade por item nas duas extrações de fatores da escala.

\begin{tabular}{|c|c|c|c|c|}
\hline \multirow{2}{*}{ Momento de leitura } & \multirow{2}{*}{\multicolumn{2}{|c|}{ Itens }} & \multicolumn{2}{|c|}{ Classificação } \\
\hline & & & 4 fatores & 3 fatores \\
\hline \multirow[t]{5}{*}{ Antes da leitura } & 1 & Imaginar como será a história lendo o título & 0,306 & 0,306 \\
\hline & 2 & Dar uma olhada na quantidade de páginas & 0,473 & 0,471 \\
\hline & 3 & Decidir se vale a pena ler a história & 0,370 & 0,215 \\
\hline & 4 & Lembrar o que já sei sobre o assunto da história & 0,340 & 0,218 \\
\hline & 5 & Ver como é a seqüência da história & 0,350 & 0,308 \\
\hline \multirow[t]{9}{*}{ Durante a leitura } & 6 & Parar de ler e pensar sobre a história para ver se estou entendendo & 0,266 & 0,239 \\
\hline & 7 & Ler novamente partes da história quando não entendo & 0,492 & 0,400 \\
\hline & 8 & Observar as gravuras para entender melhor & 0,475 & 0,450 \\
\hline & 9 & Ler de novo uma parte quando me distraio & 0,569 & 0,541 \\
\hline & 10 & Usar marca-texto para destacar o que acho importante & 0,437 & 0,403 \\
\hline & 11 & Ler com atenção e devagar para ter certeza que estou entendendo & 0,360 & 0,353 \\
\hline & 12 & Procurar no dicionário palavras novas & 0,473 & 0,466 \\
\hline & 13 & Ler em voz alta quando o texto é difícil & 0,436 & 0,255 \\
\hline & 14 & Lembrar dos principais trechos da história & 0,472 & 0,319 \\
\hline \multirow[t]{3}{*}{ Após a leitura } & 15 & Reler o texto várias vezes quando tenho dificuldade para entendê-lo & 0,509 & 0,423 \\
\hline & 16 & Relembrar os principais pontos da história para verificar se entendi & 0,448 & 0,377 \\
\hline & 17 & Conversar com meus colegas sobre as histórias para ver se entendi & 0,344 & 0,322 \\
\hline
\end{tabular}


proposta inicial de três categorias seria aprofundar a análise com extração de itens, considerando três fatores que explicam 35,70\% da variância total da escala. Apesar de quatro fatores explicarem uma variância total maior (41,89\%), os itens apresentavam-se dispersos nos fatores. Além disso, alguns deles apresentavam cargas fatoriais muito parecidas em dois dos três fatores.

Pode-se identificar que houve alteração dos valores das comunalidades para abaixo de 0,30, quando da definição de três fatores para extração, para os itens 3 e 4 relativos a antes da leitura. O item 6 que se refere a durante a leitura continuou com comunalidade inferior a 0,30 e o item 13 passou a ter valor de 0,25. Os demais itens permaneceram iguais ou tiveram alterações pequenas em seus valores (Tabela 1).

Na análise da matriz completa de todos os itens com rotação varimax, considerando três fatores, fol possível verificar a confirmação dos itens (3, 4, 6 e 13) indicados para exclusão. Esses apresentaram cargas fatoriais abaixo de 0,30 e se distribuíam em dois fatores, indicando a pouca pertinência do item à escala.

A Tabela 2 apresenta a escala com a extração dos itens indicados (3, 4, 6 e 13) e os demais itens com suas respectivas cargas fatoriais e comunalidades distribuídas pelos três fatores.

Pode-se observar que todos os itens ficaram com valor de comunalidade mínima de 0,287 e máxima de 0,561 , indicando que todos pertencem à escala. 0

Tabela 2. Itens distribuídos por fatores com valor de carga fatorial e comunalidade.

\begin{tabular}{|c|c|c|c|c|c|}
\hline $\begin{array}{l}\text { Momento } \\
\text { de leitura }\end{array}$ & Itens & & Fatores & & Comunalidade \\
\hline \multirow[t]{4}{*}{ Antes } & & Fator 1 & Fator 2 & Fator 3 & 0,323 \\
\hline & 1 & 0,560 & & & 0,561 \\
\hline & 2 & & & 0,737 & 0,287 \\
\hline & 5 & & & 0,333 & 0,467 \\
\hline \multirow[t]{7}{*}{ Durante } & 7 & & 0,670 & - & 0,453 \\
\hline & 8 & & & 0,660 & 0,589 \\
\hline & 9 & & 0,766 & & 0,464 \\
\hline & 10 & 0,676 & & & 0,328 \\
\hline & 11 & & 0,536 & & 0,504 \\
\hline & 12 & 0,689 & & & 0,323 \\
\hline & 14 & & 0,366 & & 0,419 \\
\hline \multirow[t]{3}{*}{ Após } & 15 & 0,543 & & & 0,386 \\
\hline & 16 & & 0,385 & & 0,377 \\
\hline & 17 & 0,488 & & & 0,323 \\
\hline
\end{tabular}

item Dar uma olhada na quantidade de páginas, apesar de comunalidade inferior a 0,30, possui a maior carga fatorial da escala. As cargas fatoriais também estão altas, variando de 0,333 a 0,766. Os fatores 1 e 2 ficaram com cinco itens e o fator 3 com três. Tal distribuição, dos fatores 1 e 2, apesar de numericamente desigual em relação ao 3, é aceita pela natureza das estratégias que compõem o fator 3 . As estratégias dividiram-se em fator $1(1,10,12,15$ e 17), que conta com as estratégias de apoio à leitura, sendo, pois, denominado de estratégias metacognitivas de suporte à leitura; o fator $2(7,9,11,14$ e 16) concentrou os itens relativos a estratégias usadas para resolver problemas de compreensão e foi denominado de estratégias metacognitivas de solução de problemas; e o fator 3 (2, 5 e 8) agrupou as estratégias utilizadas para uma análise geral do texto e passou a ser identificado como estratégias metacognitivas globais de leitura.

Assim, pode-se considerar que os 13 itens que passaram a compor a Escala de Estratégias de Leitura foram os que revelaram ter uma representatividade maior no instrumento em função não somente de seus valores de comunalidade e carga fatorial, mas também porque os três fatores identificados pela análise fatorial com rotação varimax explicam 42,12\% da variância da escala, assinalando um aumento nesse valor após a extração de quatro itens.

Identificou-se que os fatores estão correlacionados entre si e com o total. Verificaram-se também correlação significativa entre os momentos de leitura e uma correlação alta entre os momentos de leitura e cada fator. Esses índices também justificam a escolha do método de extração com rotação varimax e a organização dos itens nos três fatores (Tabela 3).

No geral, considerando-se a análise fatorial realizada e a presença dos três fatores determinada pelo valor da carga fatorial e do índice de comunalidade, alguns itens foram classificados de modo diferente da organização teórica inicialmente proposta e descrita na caracterização do instrumento no presente estudo (Tabela 4).

Pode-se observar que os itens extraídos referiam-se a estratégias globais utilizadas antes e durante a leitura. Houve, além disso, uma redefinição da classificação por tipo (Tabela 4). Considerando-se que os leitores iniciantes precisam das estratégias mais 
Tabela 3. Correlação entre as estratégias de leitura por momento de leitura, fator e escore total.

\begin{tabular}{|c|c|c|c|c|c|c|c|c|}
\hline \multirow{2}{*}{ Estratégias } & & \multicolumn{3}{|c|}{ Momento de leitura } & \multicolumn{3}{|c|}{ Fator } & \multirow{2}{*}{ Total } \\
\hline & & Antes & Durante & Após & Suporte & Solução & Geral & \\
\hline \multirow[t]{2}{*}{ Momento de leitura } & Antes & 1 & $0,381^{*}$ & $0,386^{*}$ & $0,481^{*}$ & $0,331^{*}$ & $0,785^{*}$ & $0,639 *$ \\
\hline & Durante & - & 1 & $0,597^{*}$ & $0,730^{*}$ & $0,829^{*}$ & $0,431^{*}$ & $0,873^{*}$ \\
\hline \multirow[t]{4}{*}{ Fator } & Após & - & - & 1 & $0,782^{*}$ & $0,630^{*}$ & $0,309^{*}$ & $0,786^{*}$ \\
\hline & Suporte & - & - & - & 1 & $0,472^{*}$ & $0,276^{*}$ & $0,822^{*}$ \\
\hline & Solução & - & - & - & - & 1 & $0,283^{*}$ & $0,770^{*}$ \\
\hline & Geral & - & - & - & - & - & 1 & $0,567^{*}$ \\
\hline
\end{tabular}

*Correlação de Pearson significante a $p<0,001$.

Tabela 4. Classificação teórica e fatorial dos itens por momento de leitura.

\begin{tabular}{|c|c|c|c|c|}
\hline \multirow{2}{*}{ Momento de leitura } & & \multirow{2}{*}{ Itens } & \multicolumn{2}{|c|}{ Classificação } \\
\hline & & & Teórica & Fatorial \\
\hline \multirow[t]{5}{*}{ Antes } & 1 & Imaginar como será a história lendo o título & global & suporte \\
\hline & 2 & Dar uma olhada na quantidade de páginas & global & global \\
\hline & 3 & Decidir se vale a pena ler a história & global & extraído \\
\hline & 4 & Lembrar o que já sei sobre o assunto da história & global & extraído \\
\hline & 5 & Ver como é a seqüência da história & global & global \\
\hline \multirow[t]{9}{*}{ Durante } & 6 & Parar de ler e pensar sobre a história para ver se estou entendendo & global & extraído \\
\hline & 7 & Ler novamente partes da história quando não entendo & global & sol. problema \\
\hline & 8 & Observar as gravuras para entender melhor & global & global \\
\hline & 9 & Ler de novo uma parte quando me distraio & global & sol. problema \\
\hline & 10 & Usar marca texto para destacar o que acho importante & sol. problema & suporte \\
\hline & 11 & Ler com atenção e devagar para ter certeza que estou entendendo & suporte & sol. problema \\
\hline & 12 & Procurar no dicionário palavras novas & suporte & suporte \\
\hline & 13 & Ler em voz alta quando o texto é difícil & suporte & extraído \\
\hline & 14 & Lembrar dos principais trechos da história & global & sol. problema \\
\hline \multirow[t]{3}{*}{ Após } & 15 & Reler o texto várias vezes quando tenho dificuldade para entendê-lo & sol. problema & suporte \\
\hline & 16 & Relembrar os principais pontos da história para verificar se entendi & sol. problema & sol. problema \\
\hline & 17 & Conversar com meus colegas sobre as históriasa ver se entendi & suporte & suporte \\
\hline
\end{tabular}

Sol.= solucionar.

como apoio e recurso para resolver problemas de compreensão, é de se esperar que os itens do instrumento em análise para as crianças que freqüentam de $2^{2}$ a $4^{a}$ série do ensino fundamental refiram-se, em maior número, às estratégias desse tipo (Dias et al. 1995; Ferreira \& Dias, 2002; Pearson \& Camperell, 2001).

Faz-se necessário também ressaltar que devido à ausência de instrumentos de avaliação de estratégias de leitura no Brasil, a categorização das estratégias de leitura desse instrumento baseou-se, principalmente, no modelo americano desenvolvido por Mokhtari e Reichard (2002). Portanto, é possível levantar a hipótese de uma não adequação dessas categorias à amostra brasileira utilizada para esse trabalho. Cabe lembrar que tal estudo contou com 1259 sujeitos, sendo 39,8\% pertencentes à escola particular e 61,2\% a escolas públicas, o que significa uma boa representatividade de sujeitos por item do instrumento (Pasquali,1999).

Em relação à fidedignidade do instrumento final, o coeficiente Alpha de Crombach indicou uma boa precisão do instrumento $(\alpha=0,73)$. Na análise de confiabilidade de Guttman split-half o coeficiente apresentado $(0,73)$ também confirma a precisão do instrumento, sendo a correlação entre as metades de 0,60 (metade $1 \mathrm{com} 7$ itens $=0,50$ e metade $2 \mathrm{com} 6$ itens $=0,65)$.

A Anova por idade e série indicou diferença muito significativa para utilização das estratégias de 
leitura por série $(f(2,1256)=3,297 ; p=0,000 ; \alpha=0,05)$. Para a idade não se constataram diferenças significativas $(f(6,1252)=1,108 ; p=0,314 ; \alpha=0,05)$ neste estudo.

O teste " $t$ " de Student para amostras independentes revelou efeito do gênero para a freqüência total de uso da escala de estratégias de leitura $(t=(1258)=5,266 ; p=0,000)$, lembrando-se que houve homogeneidade quanto ao número de participantes por gênero (51\% do gênero masculino e 49\% do feminino). As meninas $(M=21,58 ; D P=5,29)$ revelaram maior uso das estratégias que os meninos ( $M=19,96$; $D P=5,54)$. Não se identificou referência a esse resultado na literatura científica pertinente.

\section{Considerações Finais}

É relevante considerar que a análise fatorial da escala e a determinação dos três fatores equivalem à estrutura do instrumento (MARSI) desenvolvido por Mokhtari e Reichard (2002) e à Escala de Estratégias de Leitura - formato universitário (Joly, Cantalice \& Vendramini, 2004) - e consistem em uma evidência de validade de construto. Além disso, a identificação do efeito das variáveis gênero e série escolar freqüentada pelo participante são evidências de validade de critério da escala de acordo com os padrões de testagem psicológica e educacional estabelecidos pela American Educational Research Association (AERA, APA \& NCME, 1999). Faz-se necessário investigar, em estudos futuros, a relação da escala com rendimento acadêmico, o que pode vir a fortalecer tal evidência.

Sugere-se que novos estudos sejam realizados para verificar outras relações das estratégias de leitura, como, por exemplo, com desempenho em compreensão, vocabulário, inteligência, motivação, dentre outros, considerando-se a importância e a amplitude da leitura como habilidade. Além disso, é essencial que instrumentos com qualidades psicométricas sejam acrescidos aos já disponíveis para avaliação psicológica, especialmente no contexto da Psicologia Escolar e Educacional.

\section{Referências}

AERA, APA, \& NCME. (1999). Standards for educational and psychological testing. Washington: American Educational Research Association.
Brown, R., Pressley, M., Van Meter, P., \& Shuder, T. (1996). A quasi-experimental validation of transactional strategies instrucion with low-achieviment second-grade readers. Journal of Educational Psychology, 88 (1), 18-37.

Collins, M. (1998). Young children's reading estrategies. Australian Journal of Language and Literacy, 2 (1),55-64.

Dembo, M. H. (2000). Motivation and learning strategies for college sucess: a self-management approach. Mahwah: Lawrence Erlbaum Associates Publishers.

Dias, M. G. B. B., Morais, E. P. M., \& Oliveira, M. C. N. P. (1995). Dificuldades na compreensão de textos: uma tentativa de remediação. Arquivos Brasileiros de Psicologia, 47 (1), 13-24.

Ferreira, S. P. A., \& Dias, M. G. B. B. (2002). Compreensão de leitura: estratégias de tomar notas e da imagem mental. Psicologia Teoria e Pesquisa, 18 (1), 51-62.

Flavell, J. H. (1979). Metacognition and cognitive monitoring: a new area of cognitive-developmental inquiry. American Psychologist, 34 (10), 906-911.

González Fernández, A. (1992). Estrategias metacognitivas en la lectura. Madrid: Universidad Complutense.

Guthrie, J., \& Wigfield (1999). How motivation fits into a science of reading. Scientific Studies of Reading, 3 (3), 199-295.

Harp, B. (2000). The handbook of literacy assessment and evaluation (2nd ed.). Norwood: Christopher-Gordon Publishers

Hill, B. C., Ruptic, C., \& Norwick, L. (1998) Classroom based assessment. Norwood: Christopher-Gordon Publishers.

Joly, M. C. R. A. (2004). Escala de Estratégias de Leitura-nivel fundamental I. Relatório de pesquisa. Itatiba: Universidade São Francisco.

Joly, M. C. R. A., Cantalice, L. M., \& Vendramini, C. M. M. (2004). Evidências de validade de uma escala de estratégias de leitura para universitários. Interação em Psicologia, 8 (2), 261-270.

Joly, M. C. R. A., \& Noronha, A. P. P. (no prelo, 2006). Reflexões sobre a construção de instrumentos psicológicos informatizados. In A. P. P. Noronha, A. A. A. Santos \& F.F. Sisto (Orgs.)

Juel, C. (1980). Comparison of word identification strategies with varyung context, word type and reader skill. Reading Research Quartelly, 15 (3), 358-376.

Juliebo, M., Malicky, G. V., \& Norman, C. (1998). Metacognition of young readers in an early intervention. Journal of Research in Reading, 21 (1), 12-24.

Kopcke Filho, H. (1997). Estratégias para desenvolver a metacognição e a compreensão de textos teóricos na universidade. Psicologia Escolar e Educacional, 1 (2 e 3), 59-67.

Kopcke Filho, H. (2001). Estratégias em compreensão da leitura: conhecimento e uso por professores de língua portuguesa. Tese de doutorado não-publicada, Universidade de São Paulo.

Lorch, R. F., Lorch, E. P., \& Klusewitz, M. A. (1993). College student's conditional knowledge about reading. Journal of Educational Psychology, 85 (2), 239-252. 
McIntyre, E. (1990). Young children's reading strategies as they read self-selected books in schoool. Early Childhood Research Quartely, 5 (2), 265-277.

Mohktari, K., \& Reichard C. A. (2002). Assessing student's metacognitive awareness of reading strategies. Journal of Educational Psychology, 94 (2), 249-259.

Morles, A. (1986). Entrenamiento en el uso de estrategias para comprender la lectura. Lectura y Vida, 7 (1), 15-20.

Noronha, A. P. P. (2002). Os problemas mais graves e mais freqüentes no uso dos testes psicológicos. Psicologia: Reflexão e Crítica, 15 (2),135-142.

Palincsar, A., \& Brown, A. (1984). Reciprocal teaching of comprehension monitoring activities. Cognition and Instruction, 1 (2), 117-175.

Pasquali, L. (1999). Instrumentos psicológicos: manual prático de elaboração. Brasília: LabPAM/IBAPP.

Pearson, P. D., \& Camperell, K. (2001). Comprehension of text structures. In R. B. Ruddell, M. R. Ruddell \& H. Singer (Orgs.), Theorical Models and Processes of Reading (pp.448-468). Newark: Internacional Reading Association.

Pinnell, G., Lyons, C., DeFord, D., Bryk, A., \& Seltzer, M. (1994). Comparing instructional models for the literacy education of high-risk graders. Reading Research Quartely, 29 (1) , 9-39.

Rhoder, C. (2002). Mindful reading: strategy training traht facilitates transfer. Journal of Adolescent \& Adult Literacy, 45, 498-512.

Ruíz Bolívar, C. (2002). Mediación de Estratégias Metacognitivas em Tareas Divergentes y Transferencia Recíproca. Investigación y Postgrado, 17, 2. Acessado em agosto 12, 2005, disponíble en http://www.scielo.org. ve/scielo.php?
Schmidt, M. C. (2000). A questionnaire to measure children's awareness of strategic reading process. In S. J. Barrentine. Reading Assessment (pp.189-198). Newark: IRA.

Song, M. (1998). Teaching reading strategies in an ongoing EFL university reading classroom. Asian Journal of English Language Teaching, 8(1), 41-54.

Sulzby, E. (1985). Children's emergent reading of favourite storybooks: a developmental study. Reading Research Quartely, 20 (4), 458-481.

Taylor, N., Wade, M., \& Yekovich, F. (1985) The effects on text manipulation and multile reading strategies of good and poor readers. Reading Research Quartely, 20 (4), 566574.

Veenman, M. V. J., Wilhelm, P., \& Beishuizen, J. J. (2004). The relation between intellectual and metacognitive skills from a developmental perspective. Learning and Instruction, 14 (1), 89-109.

Vicentelli, H. (1999). Problemática de la lectura en estudiantes universitarios. Psicologia Escolare Educacional, 3 (3), 195-202.

Williams, J. P. (2002). Reading comprehension strategies and teacher prepation. In A. E. Farstrup \& S. J. Samuels (Orgs.), What research has to say about reading instruction (3nd ed., pp.243-260). Newark: International Reading Association.

Recebido em: 29/6/2005

Versão final reapresentada em: 28/11/2005

Aprovado em: 30/12/2005 